\title{
Homocysteine downregulates gene expression of heme oxygenase-1 in hepatocytes
}

\author{
Xiaoqin Luo ${ }^{1,2,3}$, Lei Xiao ${ }^{1}$, Haixia Yang ${ }^{1,2,3}$, Ruijuan Zhang ${ }^{2,3}$, Manli Jiang ${ }^{1}$, Jiahua $\mathrm{Ni}^{1}$, Ting Lei $^{1}$ and Nanping Wang ${ }^{1, *^{*}}$
}

\begin{abstract}
Background: Hyperhomocysteinemia (HHcy) is an independent risk factor for liver diseases, such as fatty liver and hepatic fibrosis. However, the mechanisms underlying this pro-oxidative effect of homocysteine (Hcy) in hepatocytes remain largely unknown. Thus, we investigated the effect of Hcy on the gene expression of heme oxygenase-1 (HO-1), the primary rate-limiting enzyme in heme catabolism and a key anti-oxidant detoxification enzyme in maintaining cellular redox homeostasis.

Methods: In vivo, twenty male C57BL/6 mice at 8 weeks of age were randomly divided into two groups. One group was fed a chow diet (chow group; $n=10$ ), the other group of mice was fed a methionine-supplemented diet (Met group, $1 \mathrm{mg} \mathrm{kg}^{-1}$ day $^{-1} \mathrm{~L}$-methionine in drinking water; $\mathrm{n}=10$ ) for 4 weeks. In vitro, HepG2 cells were stimulated with different doses of homocysteine (Hcy).

Results: Four weeks' methionine supplementation caused a significant increase of plasma Hcy concentration and a decrease of HO-1 expression in the liver of C57BL/6 mice than mice received chow diet. Besides, SOD enzyme activities were impaired and the level of oxidative stress markers, such as malondialdehyde (MDA) were elevated in the liver from mice supplemented with methionine compared with control mice. In cultured hepatocytes, Hcy treatment reduced both the mRNA and protein levels of HO-1 dose-dependently. However, Hcy had no effect on the gene expression of Nrf2, the major transcriptional regulator of HO-1. Instead, Hcy induced the expression of Bach1, a transcriptional repressor of HO-1. In addition, Hcy stimulated the nuclear localization of Bach1 but prevented that of Nrf2. Furthermore, we found that knockdown of Bach1 attenuated the suppression of the HO-1 expression by Hcy.
\end{abstract}

Conclusions: Collectively, our results demonstrated that Bach1 plays an important role in Hcy-triggered ROS generations through inhibiting HO-1 expression, likely, resulting from the disturbed interplay between Bach1 and Nrf2.

Keywords: Homocysteine, Reactive oxygen species, Heme Oxygenase-1, Nrf2, Bach1

\section{Background}

Homocysteine (Hcy) is a critical intermediate of methionine metabolism and has profound importance in health and diseases [1]. Indeed, the metabolism of Hcy mainly takes place in liver and normal concentrations of total Hcy in human plasma are 5-16 $\mu \mathrm{mol} / \mathrm{L}$ [2]. The disrupted metabolism of Hcy causes hyperhomocysteinemia (HHcy). There are three ranges of HHcy: moderate $(16-30 \mu \mathrm{mol} / \mathrm{L})$, intermediate $(31-100 \mu \mathrm{mol} / \mathrm{L})$, and severe $(>100 \mu \mathrm{mol} / \mathrm{L})$ [3]. Epidemiological studies have demonstrated that HHcy is an independent risk factor for

\footnotetext{
* Correspondence: nanpingwang2003@yahoo.com

${ }^{1}$ Cardiovascular Research Center, School of Medicine, Xi'an Jiaotong University, Xi'an 710061, China

${ }^{4}$ Institute of Cardiovascular Science, Peking University, Beijing 100191, China Full list of author information is available at the end of the article
}

fatty liver diseases, cardiovascular disease, diabetes and so on [3-7]. Clinical and experimental studies have shown that pathophysiological effects of Hcy involved the excessive generation of reactive oxygen species (ROS) [8-10]. However, the molecular mechanisms underlying the effect of Hcy on oxidative stress have not been explored.

Heme oxygenase- 1 (HO-1), the primary rate-limiting enzyme in heme catabolism, acts as a key anti-oxidant detoxification enzyme in maintaining cellular redox homeostasis [11]. HO-1 is involved in many pathophysiological changes of liver injuries including liver oxidative stress, chronic inflammation and so on. It has been reported that the induction of HO-1 as well as its reaction products can protect the liver against damage caused by a number of chemical compounds [12-16]. Otherwise, 
HO-1 knockout mice developed major hemosiderosis and chronic inflammatory in liver, suggesting HO-1 induction as an important cellular endeavor for hepatoprotection $[17,18]$. The primary mechanism for up-regulation of the HO-1 gene is by increasing transcription of this gene $[19,20]$. This process is mediated by binding of nuclear factor erythroid 2-related factor 2 (Nrf2), a basic leucine zipper transcription factor, to antioxidant response element (ARE) sequence [21]. In the presence of oxidative stimuli, activated Nrf2 translocates into the nucleus, binds to the heme-responsive elements (HeRE) in the $5^{\prime}$-UTR of the HO-1 promoter, and commences transcription of HO-1 [22,23]. However, the regulation of HO-1 in HHcy has not been well studied. Therefore, we examined how Hcy modulates the stress protein HO-1 in hepatocytes. In the current study, we demonstrated that Nrf2 as well as Bach1 are involved in the downregulation of $\mathrm{HO}-1$ gene expression by Hcy. The subcellular localisation of these two transcription factors plays critical roles in this regulating effect.

\section{Methods}

Animal experiments

The animal experiments conform to the Guide for the Care and Use of Laboratory Animals that was published by the US National Institute of Health (NIH Publication No. 8523, revised 1985). Male C57BL/6 mice at 8 weeks of age were obtained from the Experimental Animal Center of Xi'an Jiaotong University (Xi'an, China) and fed with either chow diet $(n=10)$ or methionine-rich diet $\left(1 \mathrm{mg} \mathrm{kg}^{-1}\right.$ day $^{-1}$ L-methionine in drinking water; $\mathrm{n}=10$ ) for 4 weeks. Each mouse was separately housed in a temperature-controlled $\left(24^{\circ} \mathrm{C}\right)$ facility with a $12 \mathrm{~h}$ light/ $12 \mathrm{~h}$ dark cycle with free access to food and autoclaved water. We weighed the mice every week and calculated the consumption of water and food for each group. After being anaesthetized by intraperitoneal injection of $3 \%$ chloral hydrate, the blood of mice were collected from the orbit. The study and all of the procedures were approved by the Xi'an Jiaotong University Animal Experiment Committee.

\section{Plasma Hcy determination}

Plasma Hcy concentrations were measured as previously described [24]. Briefly, the blood samples were collected in EDTA-containing tubes, which were then immediately centrifuged at 3,000 g for $10 \mathrm{~min}$. The concentrations of plasma total Hcy concentrations were quantified using a fluorescence polarization immunoassay ELISA Kit (Abbott IMx).

\section{SOD enzyme activity unit determination}

The livers were blotted dry and weighed and then homogenized in 8 volumes of lysis buffer $(\mathrm{pH} 7.4)$ at $4^{\circ} \mathrm{C}$ for 30 seconds using a polytron homogenizer. The homogenate was centrifuged at $12,000 \times \mathrm{g}$ for $15 \mathrm{~min}$, and then was used to detect the activity units of SOD enzyme by a commercial kit (Biovision).

\section{Determination of Malondialdehyde (MDA)}

The protein concentration of the supernatants of liver homogenate was determined using the Pierce ${ }^{\mathrm{T} M}$ BCA Protein assay kit (Thermo Fisher Scientific Inc., Rockford, USA) according to the user manual. The MDA concentrations were detected with a kit according to the manufacturer's instructions (R\&D Systems, Inc., USA) and normalized to protein levels.

\section{Cell culture}

HepG2 cells (ATCC, Manassas, VA, USA) were cultured with Dulbecco's Modified Eagle's Medium (DMEM) containing $10 \%$ fetal bovine serum (FBS). Cells were seeded into 6-well plates $24 \mathrm{~h}$ prior to treatments at approximately $80 \%$ confluence and exposed to different doses of Hcy (Sigma-Aldrich, St. Louis, MO) for additional $24 \mathrm{~h}$.

\section{Quantitative reverse transcription PCR (qRT-PCR)}

The total RNA of liver homogenate and cells were obtained and qRT-PCR was conducted as previously described [25]. Primers against Bach1 (forward primer, 5'-GGACACTCCTTGCCAAATGCAG-3'; reverse primer, 5' -TGACCTGGTTCTGGGCTCTCAC-3'); Nrf2 (forward primer, 5' -AGC ACACCCAGTCAGAAACCAG-3'; reverse primer, 5' -TCTACAAACGGGAATGTCG-3'); HO-1 (forward primer, 5'-CGCTGGCAGGAGGTCAT-3'; reverse primer, $5^{\prime}$-CATCGGAGAAGCGGAGC-3') and GAPDH ((forward primer, 5' - ACCACAGTCCATGCCA TCAC-3'; reverse primer, 5' -TCCACCACCCTGTTGCT GTA-3') were designed using the sequence information of the NCBI database. The PCR conditions were as follows: initial denaturation: $95^{\circ} \mathrm{C}, 5 \mathrm{~min} ; 40$ cycles of denaturation $\left(95^{\circ} \mathrm{C}, 30 \mathrm{~s}\right)$, annealing $\left(58^{\circ} \mathrm{C}, 30 \mathrm{~s}\right)$, and elongation $\left(72^{\circ} \mathrm{C}\right.$, $60 \mathrm{~s})$. The fluorescent signals were collected during the extension phase, $\mathrm{Ct}$ values of the sample were calculated, and the mRNA levels were analyzed by $2^{-\Delta \Delta C t}$ method.

\section{Western blotting}

Proteins from liver homogenate were extracted with ice-cold lysis buffer (Roche, Mannheim, Germany). Additionally, the nuclear proteins were extracted by the Pierce NE-PER kit (Pierce, Rockford, IL, USA) according to the manufacturer's instruction. All steps were carried out on ice. Proteins concentrations were determined by the BCA assay (Pierce). Protein $(25 \mu \mathrm{g})$ from each sample was separated by $10-12 \%$ SDS-PAGE and electrotransferred to Polyvinylidene Fluoride (PVDF) membranes. The membranes were blocked with 5\% BSA for $1 \mathrm{~h}$ at room temperature and incubated overnight at $4^{\circ} \mathrm{C}$ using the 
following 1:1000 primary rabbit antibodies (Cell Signaling Technology Inc. MA, USA) of HO-1 (\#5853), Bach1 (\#4578), Nrf2 (\#12721), Histone and mouse antibody of $\beta$-actin, followed by 1:4000 dilution of goat anti-rabbit or goat anti-mouse horseradish peroxidase-labeled antibodies (Cell Signaling Technology Inc. MA, USA). The bands were visualized using the ECL system, and the band density was determined by Image J software (NIH, USA).

\section{Measurement of reactive oxygen species}

The intracellular ROS level of cells was detected by fluorescence microscope and flow cytometry using the fluorescent probe $2^{\prime}, 7^{\prime}$-Dichlorofluorescin diacetate (DCFH-DA), which is a cell-permeable non-fluorescent probe and turns to highly fluorescent 2 ', 7 ' -Dichlorofluorescin (DCF) upon oxidation. The cells were quiesced for $24 \mathrm{~h}$ and treated with different doses of $\mathrm{Hcy}$ for additional $24 \mathrm{~h}$ or $\mathrm{H}_{2} \mathrm{O}_{2}$ $(100 \mu \mathrm{M})$ for $30 \mathrm{~min}$ as a positive control. Then, the cells were washed with PBS, and incubated with $10 \mu \mathrm{M}$ DCFH-DA at $37^{\circ} \mathrm{C}$ for $30 \mathrm{~min}$ in dark. After incubation, the cells were washed with PBS and observed under fluorescent microscopy directly or adjusted to a concentration of $1 \times 10^{6} / \mathrm{mL}$ after trypsinization and analyzed by FACScan (BD FACSAriaTM III, United States) within $30 \mathrm{~min}$. 10,000 cells were counted in each determination and the specific fluorescence signals were collected with a 488-nm band pass filter.

\section{Luciferase reporter assay}

The HO-1 promoter reporter (pGL3-HO-1) was constructed. The fragment spanning from -3000 base pairs upstream of the transcription start of the human HO-1 gene was subcloned into pGL3-basic plasmid containing the ARE sequence and the firefly luciferase reporter gene (Promega Corporation, Madison, USA). The reporter plasmid and $\beta$-gal were transfected into HepG2 cells using the Lipofectamine 2000 reagents from Invitrogen according to the manufacturer's instructions. These cells were subsequently treated with different dose of Hcy (0, 100, 500, $1000 \mu \mathrm{M}$ ) for $24 \mathrm{~h}$. Cell lysates were harvested to measure the luciferase activities using the Dual-Luciferase ${ }^{\oplus}$ Reporter Assay System (Promega). The luciferase activities were then normalized to $\beta$-galactosidase activity.

\section{Gene silencing}

To knock down Bach1 expression, cells were transfected with 100 pmol Bach1 siRNA, a commercially available small interfering RNAs (siRNAs) duplex components against this molecule (sc-37064; Santa Cruz, CA, USA) or a negative control siRNA (sc-37007; Santa Cruz, CA, USA), a RNA duplex with no known sequence homology, in a 12-well format using Lipofectamine 2000 (Invitrogen, Carlsbad, CA) according to the manufacturer's instructions.

\section{Statistical analysis}

All of the experiments were repeated at least three times. Data are expressed as means \pm SEM. The significance of differences between 2 or more than 2 groups was determined by Tamhane's test and one-way ANOVA, respectively, using SPSS 16.0 software (SPSS, Chicago, IL). A value of $P<0.05$ was considered to be statistically significant.

\section{Results}

Hcy accumulation increased oxidative stress and down-regulated HO-1 expression in mouse livers

To determine the effect of Hcy on HO-1 expression, mice were supplemented with L-methionine in drinking water (Met group). After 4 weeks of treatment, the plasma Hcy concentrations significantly raised compared to those in chow diet-fed mice (chow group) (Figure 1A). It is well established that oxidative stress is implicated in Hcy-caused liver injury. Relative analysis demonstrated that SOD enzyme activity was decreased significantly to 45.9\% in Met groups compared with the Chow group mice (Figure 1B). Furthermore, the mice from Met group had higher levels of MDA in livers than chow group (Figure $1 \mathrm{C}$ ). Next, we identify the effect of elevated Hcy on the expression of $\mathrm{HO}-1$, a key anti-oxidant detoxification enzyme in maintaining cellular redox homeostasis. The mRNA levels of HO-1 were remarkably reduced in Met group than chow group (Figure 1D). Then, liver homogenate supernatants were then assayed by Western blotting. Consistently, the mice from Met group had an obvious decrease in HO-1 expression compared with chow group (Figure 1E). We also detected the effects of increased level of Hcy on the expression of Bach1 and Nrf2, which are two main regulator of HO-1. As shown in Figure 1F, Met supplementation induced Bach1 protein level whereas the expression of Nrf2 was comparable between two groups.

\section{Hcy dose-dependently induced intracellular ROS production in hepatocytes}

Next, we performed in vitro culture of HepG2 cells with Hcy. The concentrations in this study did not affect cell viability (Additional file 1: Figure S1). The effect of Hcy on intracellular ROS levels is shown in Figure 2. Cells were treated with different doses of Hcy for $24 \mathrm{~h}$ and the ROS levels were determined by flow cytometry or fluorescent microscope after incubation of the fluorescent probe DCFH-DA. Hcy increased the intracellular ROS levels in a dose-dependent manner (Figure $2 \mathrm{~A}$ and $2 \mathrm{~B}$ ). As a positive control, the treatment with $100 \mu \mathrm{M} \mathrm{H} \mathrm{H}_{2} \mathrm{O}_{2}$ for $30 \mathrm{~min}$ significantly triggered ROS generation of HepG2 cells. Similar results were obtained by fluorescent microscope. Hcy dose-dependently enhanced the intensity of fluorescence signal of DCF (Figure 2C). 

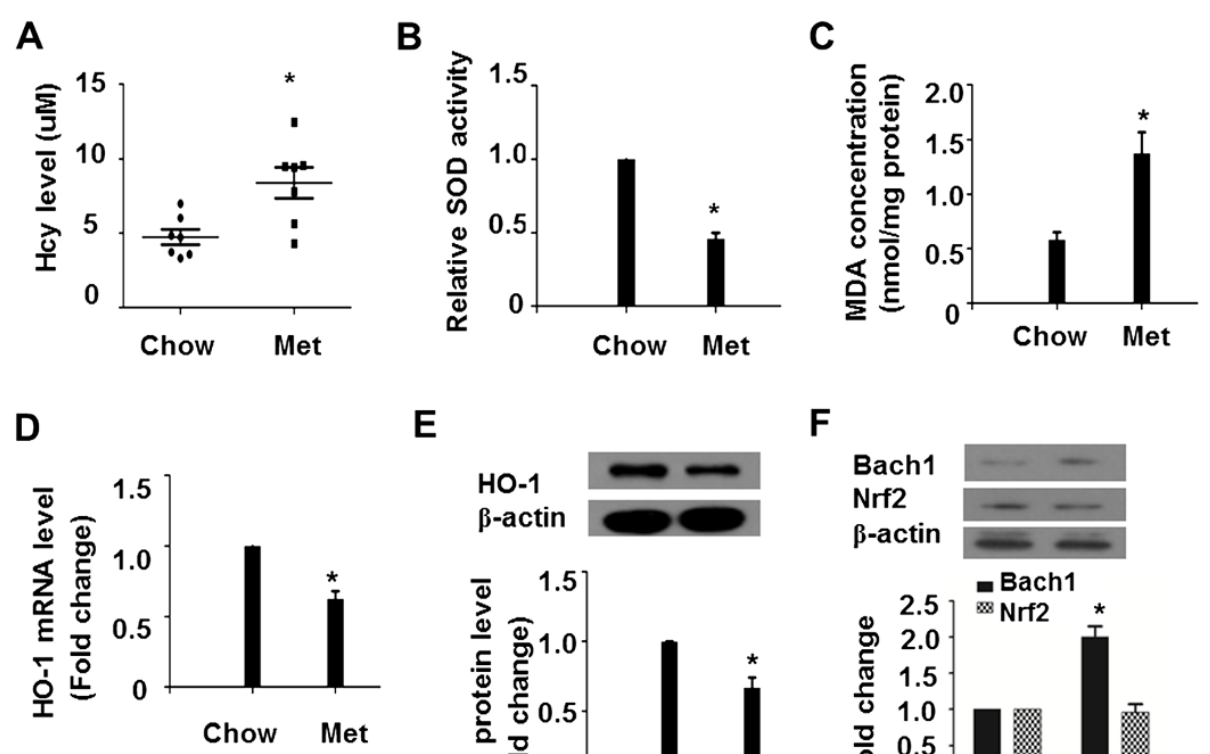

E

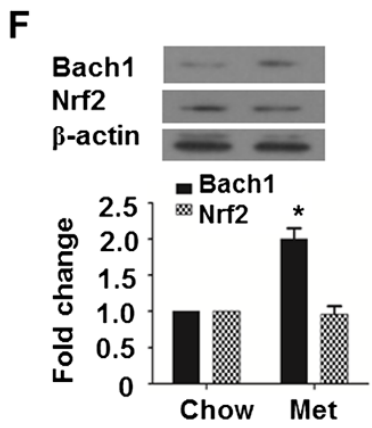

Figure 1 Hcy accumulation resulted in oxidative stress and HO-1 downregulation. (A) Hcy concentrations of plasma were determined by an ELISA assay and indicated for each group of C57BL/6 J mice ( $n=7$ for each group). The livers were homogenized and the total protein concentrations were assessed to determine the enzyme activity units of SOD by WST-1 method (B) and the MDA concentrations using an ELISA assay (C). (D) The total RNA of livers were extracted and subjected to qRT-PCR for the assessment of HO-1 mRNA level. The bar graph shows mRNA levels of HO-1 after normalization to GAPDH. Protein levels of HO-1, Bach1 and Nrf2 were analyzed using Western blotting (E and F). $\beta$-actin was used as an internal control to ensure equal loading in all lanes of the gel. Representative results of 3 independent experiments are shown. Data are presented as means \pm SEM from 3 three independent experiments. ${ }^{*} P<0.05$ vs. mice fed with chow diet.

Hcy down-regulates HO-1 expression

To determine the effect of Hcy on HO-1 expression in HepG2 cells, qRT-PCR and Western blotting were performed to detect the mRNA and protein levels of $\mathrm{HO}-1$, respectively. As shown in Figure 3A, Hcy dose-dependently decreased the HO-1 mRNA levels after $24 \mathrm{~h}$ exposure. The statistical analysis showed that HO-1 expression levels significantly differ from each other $(P<0.05)$. In order to examine whether the effect of Hcy on HO-1 mRNA expression is on a transcriptional level, we cloned a luciferase-reporter driven by the $3 \mathrm{~kb} \mathrm{HO}-1$ promoter fragment of the human HO-1 gene. The results of luciferase assays showed that Hcy inactivated the HO-1 promoter dose-dependently (Figure 3B). Next, we detected the expression of $\mathrm{HO}-1$ protein by western blotting assays. Compared with untreated cells, HO-1 protein expression declined to $98.1 \%, 54.3 \%$ and $23.7 \%$ in cells treated with Hcy at the concentrations of 100, 500 and $1000 \mu \mathrm{M}$, respectively, and the differences were statistically significant from each other (Figure 3C). We also determined the mRNA level of Nqo1 and GSTA1, other two target genes of Nrf2, and found that Hcy reduced the expression of Nqo1 dose-dependently whereas the expression of GSTA1 was only decreased in higher doses of Hcy (Additional file 1: Figure S2).
Hcy had opposite effects on the nuclear localization of Bach1 and Nrf2

Since Bach1 can down-regulate HO-1 transcription through competing with Nrf2 for the binding of antioxidant response element (ARE), the levels of nuclear and cytosolic Bach1 and Nrf2 were examined by Western blotting. The proteins in cytoplasm were shown in Figure 4A, the Nrf2 protein elevated gradually following Hcy treatment at concentrations of 100,500 and $1000 \mu \mathrm{M}$ while the fractions of Bach1 were decreased remarkably compared to those of control cells. The statistical analysis showed significant differences from each other (Figure 4C, $P<0.05)$. In contrast with the localization of the two proteins in cytoplasm, nuclear Bach1 accumulated and nuclear Nrf2 reduced dramatically after Hcy exposure at the concentrations above (Figure 4B and D).

\section{Hcy induced the expression of Bach1 but not Nrf2 in HepG2 cells}

We next evaluate the effect of Hcy on Nrf2 gene expression since $\mathrm{Nrf} 2$ is involved in cellular protection against oxidative stress through antioxidant response element (ARE)-directed induction of several phase 2 detoxifying and antioxidant enzymes, including HO-1 [26]. Interestingly, Hcy had no significant effect on Nrf2 mRNA levels. 


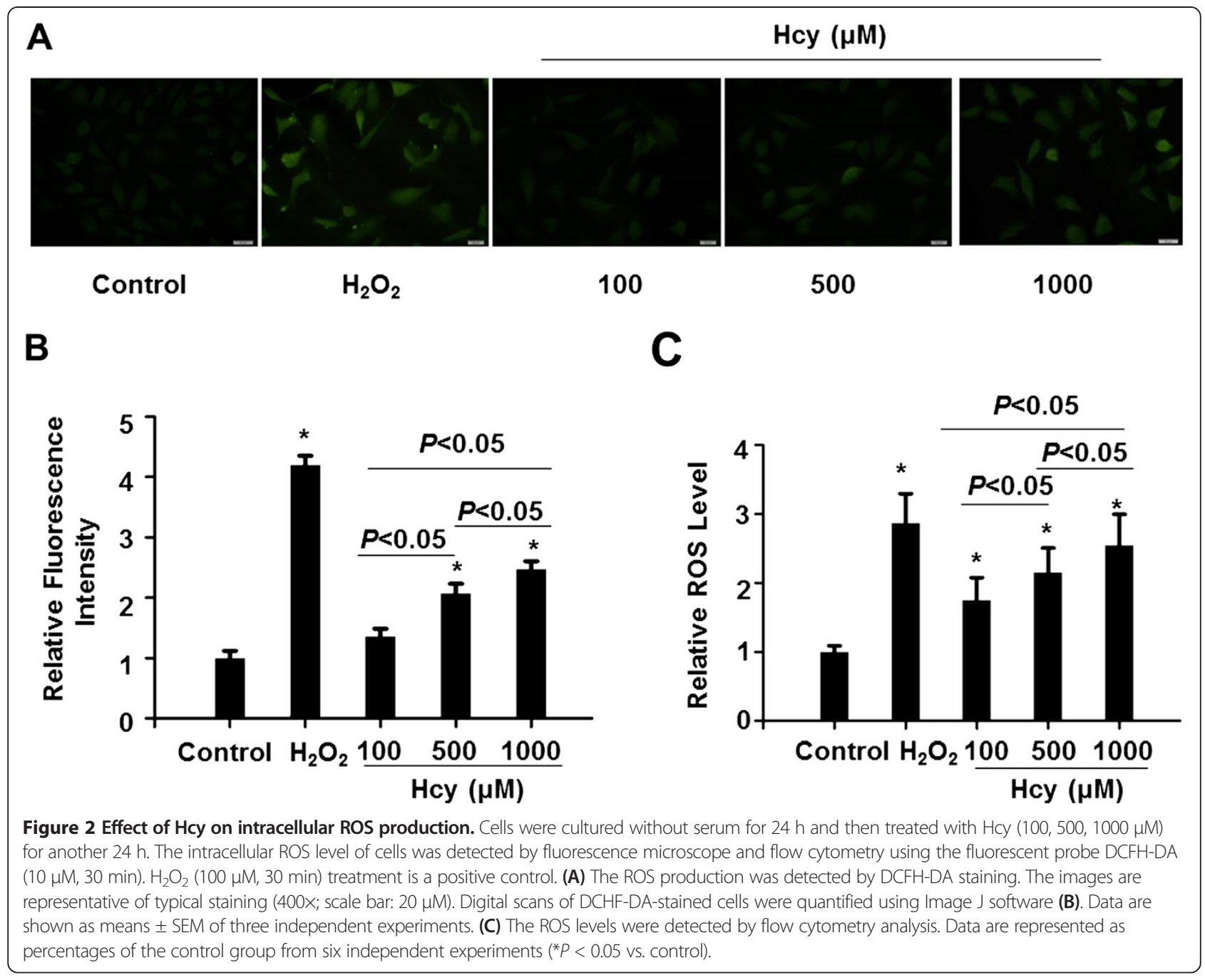

In contrast, the mRNA level of another transcription factor Bach1, which was suggested to represses the transcription of HO-1, increased in a concentration-related manner after Hcy treatment (Figure 5).

Bach1 siRNA reversed the suppression of HO-1 expression by Hcy

To determine the effect of Bach1 on the regulation of HO-1 expression in Hcy-treated HepG2 cells, we silenced human Bach1 gene expression by Bach1 siRNA. After the transfection of 100 pmol Bach1 siRNA for $72 \mathrm{~h}$, the Bach1 protein level was markedly reduced to $16 \%$ compared to those of control siRNA-treated cells (Figure 6A). The levels of HO-1 mRNA were significantly increased (37.1-fold) after exposure to Bach1 siRNA without Hcy treatment. Compared to control siRNA-treated cells, Bach1 siRNA treatment significantly reversed the suppression of HO-1 gene expression by Hcy (Figure 6B). However, Hcy failed to attenuate the expression of HO-1 significantly in Bach1 siRNA-treated cells even with a slight decrease $(\mathrm{P}=0.12,0.09$ and 0.07 for Hcy at the concentrations of 100, 500 and $1000 \mu \mathrm{M}$, respectively, when compared with Bach1 siRNA-treated cells only).

\section{Discussion}

Hyperhomocysteinemia (HHcy) is an independent risk factor for liver diseases, such as fatty liver and hepatic fibrosis [5]. The pathophysiological changes of HHcyinduced liver injuries include chronic inflammation, fibrosis, cirrhosis, and so on. In the present study, we provided novel result that Hcy decreased the expression of HO-1, a key anti-oxidant detoxification enzyme, via deregulated interplay between $\mathrm{Nrf} 2$ and Bach1, resulting oxidative stress in hepatocytes. This effect of Hcy on HO-1 is highly dose-dependent and may represent another mechanism contributing to the pro-oxidative effects of Hcy.

Nutritional factors such as high methionine-diet are associated with increased serum homocysteine levels. Extensive studies have shown that a high methionine-diet had awful effects on liver pathophysiology, for instance, 

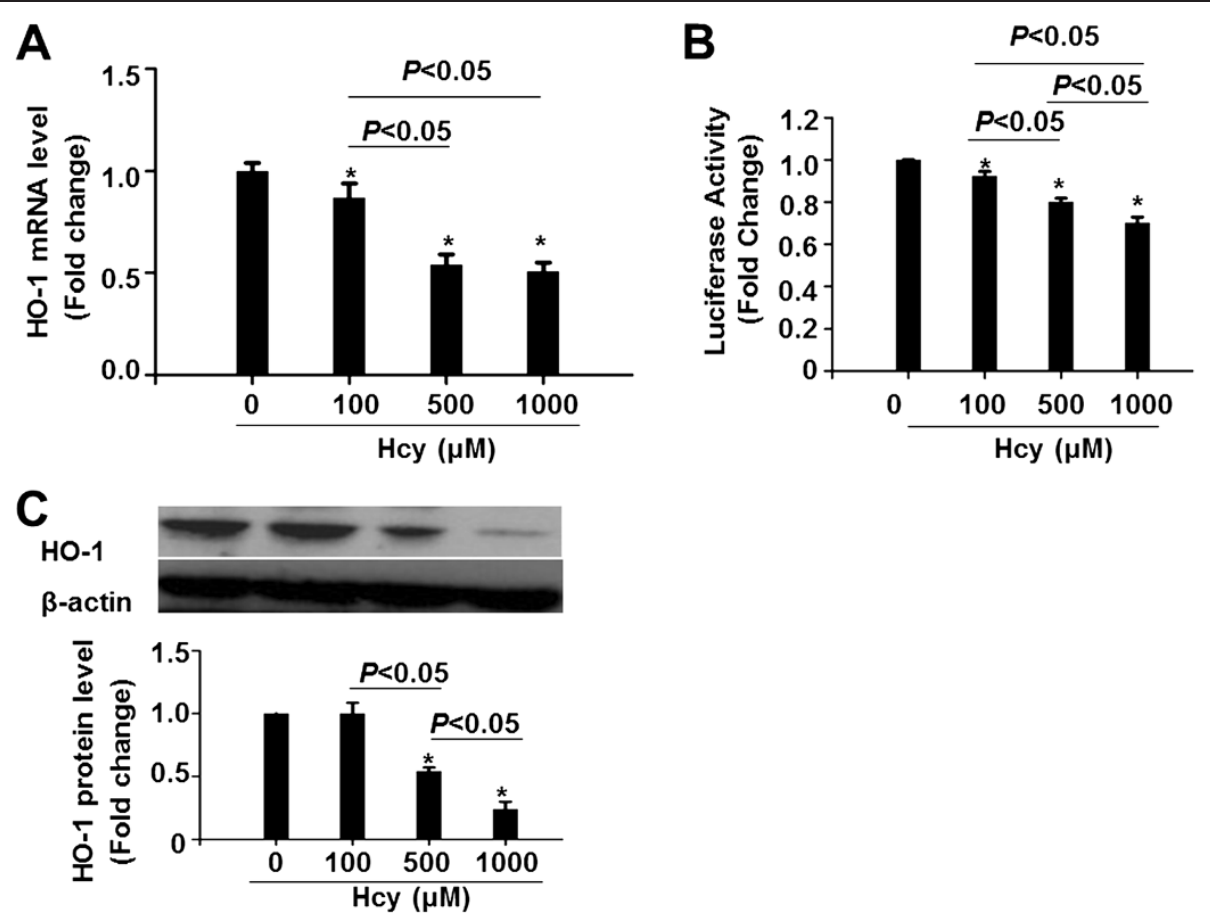

Figure 3 Hcy dose-dependently down-regulates HO-1 gene expression in HepG2 cells. HepG2 cells were treated with the indicated concentrations of Hcy for 24 h. (A) Total RNA was extracted and subjected to qRT-PCR for the assessment of HO-1 mRNA level. The bar graph shows mRNA levels of $\mathrm{HO}-1$ after normalization to GAPDH. Data are presented as means \pm SEM from 3 three independent experiments. ${ }^{*} P<0.05$ vs. control. (B) HepG2 cells were transfected with the HO-1-pGL3 plasmid and then exposed to Hcy for $24 \mathrm{~h}$. The data are expressed as fold change of the luciferase activities normalized to $\beta$-gal activity compared to control. ${ }^{*} P<0.05$ vs. control; $n=4$. (C) Protein levels of HO- 1 were analyzed using Western blotting. Representative results of three independent experiments are shown. $\beta$-actin was used as an internal control to ensure equal loading in all lanes of the gel.

oxidative stress, inflammation, lipid metabolism disorders and fibrosis $[27,28]$. Our observations are in line with previous studies reporting that methionine supplementation increased plasma Hcy levels, triggered oxidative stress of livers and impaired the anti-oxidative enzyme activity. Furthermore, methionine treatment attenuated the expression of HO-1 in the liver. Therefore, we next performed in vitro culture of hepatocytes to Hcy and found that Hcy treatment dose-dependently downregulates HO-1 expression. These results suggested that Hcy accumulation has a promoting effect on oxidative stress, which might be partially contributed by the decrease of anti-oxidative enzyme HO-1. However, whether Hcy affect the HO-1 enzymatic activity remains to be characterized and this limitation is warranted to be examined in future study.

$\mathrm{Nrf} 2$ is the main transcriptional activator of the HO-1 gene in response to various forms of stimulation such as oxidative stress [29]. Activation of the Nrf2/HO-1 pathway can protect cells from oxidative stress-induced damage [30]. Antioxidants are strong activators of Nrf2 because after metabolism they produce a small amount of oxidative stress that signals Nrf2 activation [31-33].
Inversely, chemicals with oxidant stimulation are able to repress $\mathrm{Nrf} 2 / \mathrm{HO}-1$ pathway [34-36]. In the present study, we found that Hcy which triggered ROS generation significantly inhibited HO-1 mRNA and protein levels in HepG2 cells. As for the result that Nrf2 mRNA levels did not change obviously after Hcy treatment, it is possible that a decreased binding of Nrf2 to ARE sequence is a potential mechanism as well. Firstly, the decreased nuclear translocation of Nrf2 would lead to a reduced binding to its cognate sequences in the target gene HO-1. Secondly, the transfection and ChIP assays indeed confirmed that Bach1 and Nrf2 competed with each other on the ARE sequence in HepG2 cells [37].

Like Nrf2, Bach1 is also a member of the basic leucine zipper family of proteins [38]. It was previously reported that Bach1 plays a critical role in heme-dependent downregulation of the human HO-1 gene [39,40]. Sun et al. have showed that HO-1 is constitutively expressed at higher levels in many tissues of Bach1-deficient mice, suggesting that Bach-1 acts as a negative regulator of transcription of the mouse $H O-1$ gene [41]. Here, our results demonstrated that Hcy induced Bach1 expression at the transcriptional level. Further RNA interference experiments allowed us to 

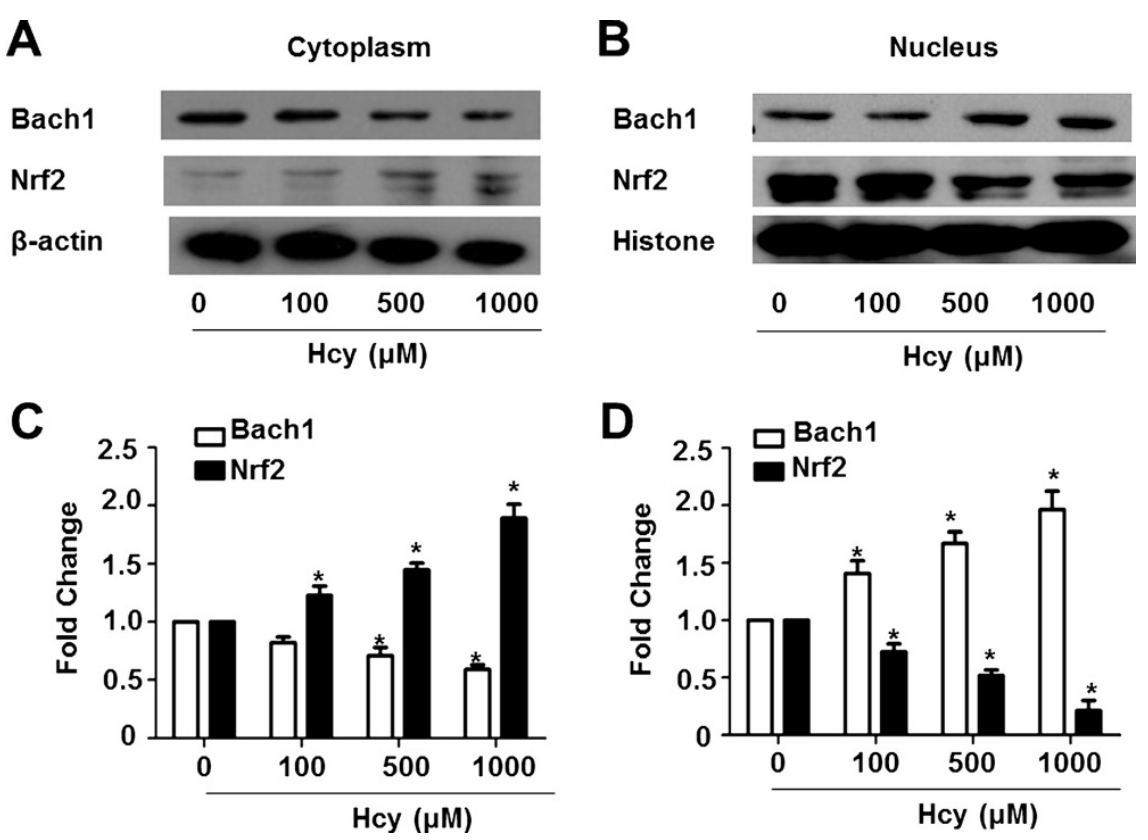

Figure 4 Hcy induced nuclear import of Bach1 and the export of Nrf2. The cytosolic and nuclear proteins were extracted separately after Hcy treatment and subjected to Western blotting assay. Representative images of three independent experiments were shown. The cytosolic (A) and nuclear (B) proteins were present to illustrate the changes of subcellular localization of Nrf2 and Bach1. The values of density of proteins were justified with $\beta$-actin (C) and Histone (D), accordingly. The relative density ratios of untreated cells were set at a value of 1.0. The values are means \pm SEM from three independent experiments. ${ }^{*} P<0.05$ vs. control.

confirm the core role of Bach1 in the suppression of HO-1 to Hcy. On the other hand, Bach1 can regulate HO-1 gene expression by competing with Nrf2 [42,43]. Under oxidative stress, Nrf2 is released from Keap1 and translocated into the nucleus. In the nucleus, Bach1 can form heterodimers with the Maf-related protein family [44],

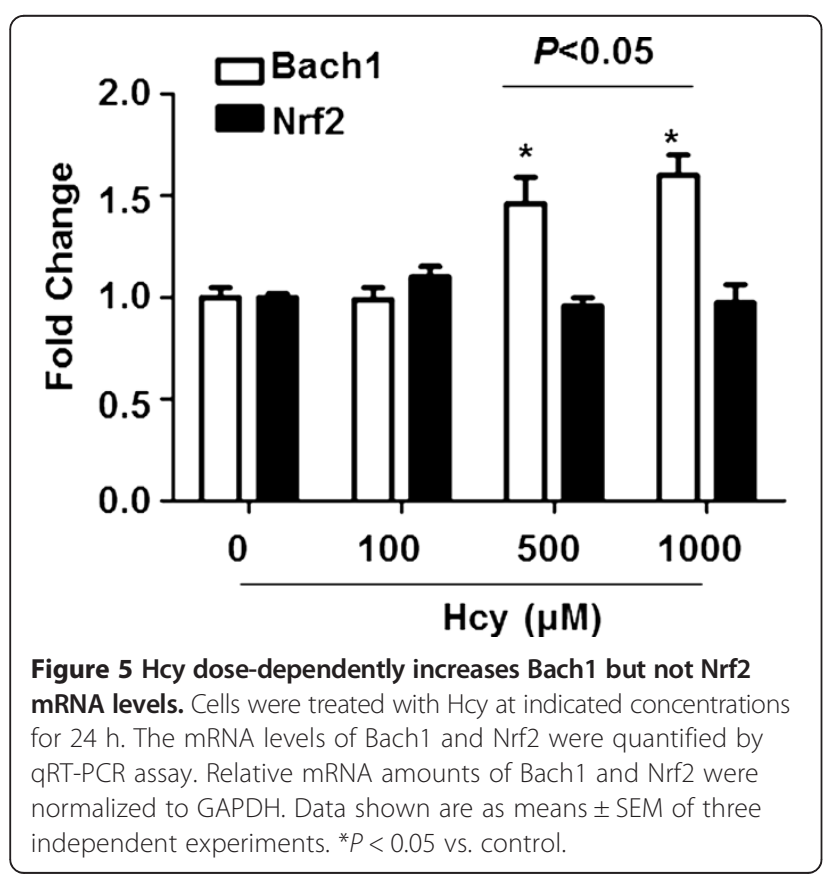

and then leads to switching off Nrf2 and suppression of cytoprotective gene expression to basal levels through competition with Nrf2 for binding to ARE [37]. Hence, the nuclear import of Bach1 is supposed to allow for unhindered nuclear export of Nrf2. As expected, we found Hcy treatment caused an alteration of nuclear localizations of Bach1 and Nrf2. Consequently, this modification process of the two transcription factors was associated with a significant decrease in the expression of the downstream cytoprotective phase 2 genes $\mathrm{HO}-1$. These results demonstrated that the increase of Bach1 gene expression and its nuclear translocation potentiated the downregulation of the HO-1 by Hcy.

A previous study has suggested that incubation of Hcy for very short time (45 $\mathrm{min}$ ) induced the expression of HO-1 in HepG2 cells [45]. It seems that the oxidative stress provoked by Hcy stimulated the immediate cellular response in HepG2 cells by activating the Nrf2-ARE pathway. However, some certain degree of maladaptation will occur when cells were exposed to higher levels of Hcy at longer duration. It has been reported that high levels of Hcy $(500 \mu \mathrm{M})$ were able to abolish hypoxiamediated HO-1 expression in a concentration-dependent manner [46]. Actually, the mechanism of signal transduction from antioxidant to Nrf2 is complex and involves basal, pre-induction, induction and post-induction phases [47]. Cellular exposure to oxidants usually firstly triggers the pre-induction response in which negative regulators of 


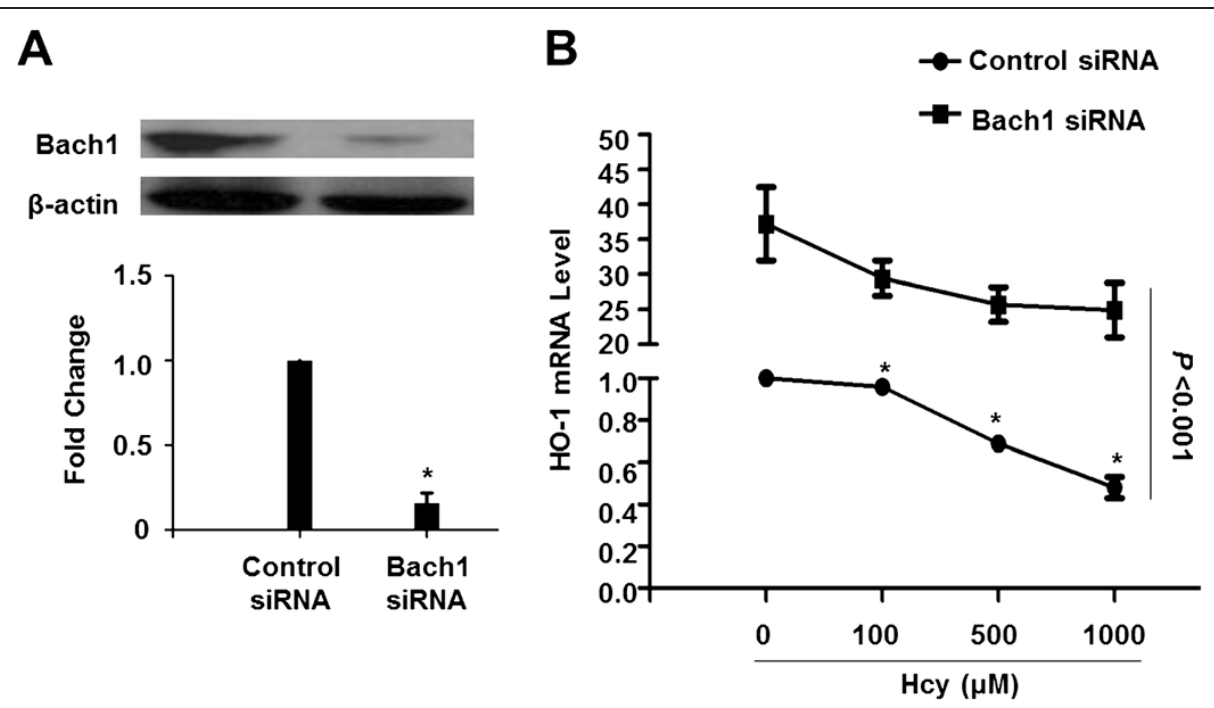

Figure 6 The effect of Bach1 on the regulation of HO-1 expression in Hcy-treated HepG2 cells. Cells were treated with 100 pmol Bach1siRNA or control siRNA for $72 \mathrm{~h}$ before Hcy treatment (0, 100, 500, $1000 \mu \mathrm{M}$ for $24 \mathrm{~h}$ ). (A) Protein level of Bach1 was analyzed using Western blotting. $\beta$-actin was used as a internal control. Representative image of three independent experiments was shown. (B) HO-1 mRNA levels were detected by qRT-PCR. Each data point represents means \pm SEM from three independent experiments. Values for cells treated with control siRNA-only were set equal to 1. The two curves are significantly different from one another using nonlinear regression analysis $(P<0.001)$ and the data points are significantly different from one another. ${ }^{*} P<0.05$ vs. cells treated with control siRNA-only.

Nrf2 are exported out of nucleus. Consequently, Nrf2 itself is activated and increase $\mathrm{HO}-1$ transcription to defense oxidative stress. However, it is worth noting that this protective benefit is limited since the induction phase Nrf2 is followed by the post-induction phase, which switches off Nrf2 activation [48].

In summary, our results showed that Hcy markedly reduces HO-1 mRNA and protein levels in hepatocytes. This down-regulation effect was associated with up-regulation of Bach1 mRNA levels. In addition, Hcy promoted the alteration of nuclear localization of Bach1and Nrf2 (Figure 7). The results of our study confirmed that the subcelluar localizations of Nrf2 and Bach1 caused by the excessive oxidative stress in Hcy-treated hepatocytes could account for the down regulation of $\mathrm{HO}-1$ expression, which may be responsive for the HHcy-induced liver injury.

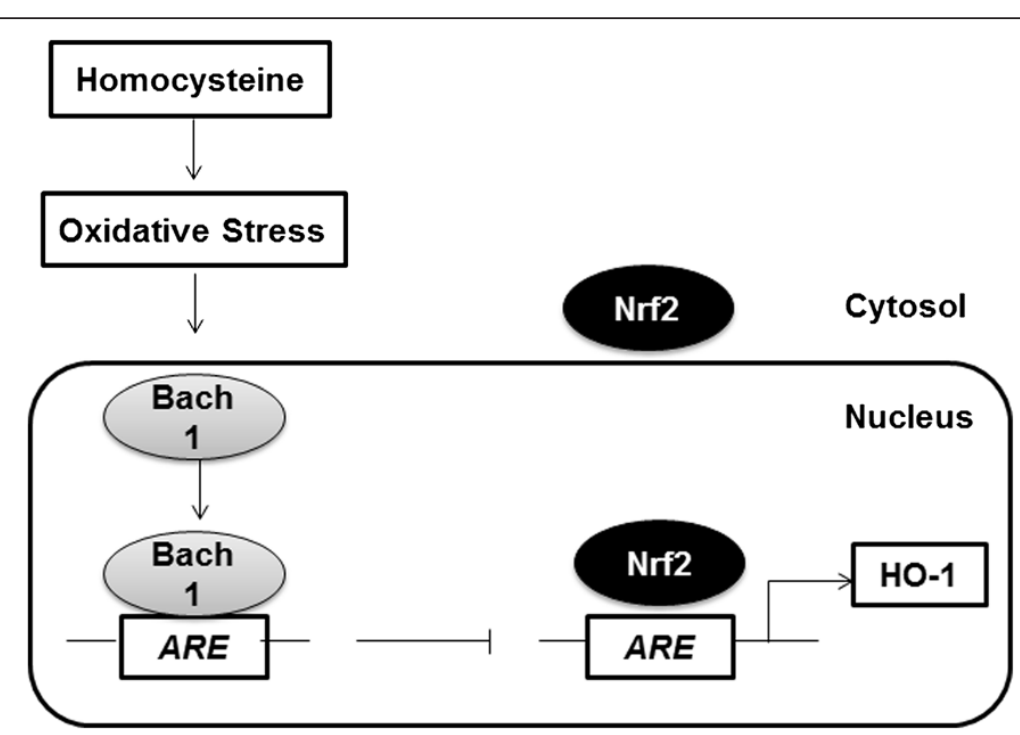

Figure 7 Schematic model of effect of Hcy on HO-1 expression via regulation of Bach1 and Nrf2. With high levels of Hcy, expression of Bach1, a repressor of HO-1 gene expression, is increased. Bach1 also competes with Nrf2 to bind antioxidant response element (ARE), then reduces $\mathrm{HO}-1$ transcription. 


\section{Additional file}

Additional file 1: Figure S1. The effect of different dose of Hcy on cell viability. After the treatment of different doses of Hcy for $24 \mathrm{~h}$, cells were incubated for additional $4 \mathrm{~h}$ in the presence of a MTT labelling mixture. The absorbance of the samples was measured at $550 \mathrm{~nm}$ with using a microliter plate (ELISA) reader. The results were presented as fold change of the absorbance against the blank compared to control. Figure S2. Hcy down-regulates gene expressions of Nqo1 and GSTA1. HepG2 cells were treated with the indicated concentrations of Hcy for $24 \mathrm{~h}$. Total RNA was extracted and subjected to qRT-PCR for the assessment of Nqo1 (A) and GSTA1 (B) mRNA levels. The bar graph shows mRNA levels of the two genes after normalization to GAPDH. Data are presented as means \pm SEM from 3 three independent experiments. ${ }^{*} P<0.05$ vs. control.

\section{Abbreviations}

Hcy: Homocysteine; ROS: Reactive oxygen species; HO-1: Heme oxygenase-1; Nrf2: Nuclear factor erythroid 2-related factor 2; ARE: Antioxidant response element; siRNA: Small interfering RNA; GAPDH: Glyceraldehyde-3-phosphate dehydrogenase; DCFH-DA: 2',7'-Dichlorofluorescin diacetate; PVDF: Polyvinylidene fluoride.

\section{Competing interest}

The authors declare that they have no competing interests.

\section{Authors' contributions}

NPW and $X Q L$ are responsible for the design of the research; $X Q L, M L J, T L$ and NJH performed the experiments; XQL and HXY analyzed the data; NPW and $X Q L$ interpreted the results of the experiments; NPW and XQL prepared the figures and drafted the manuscript; NPW, LX RJZ and XQL edited and revised the manuscript. All authors read and approved the final version of the manuscript.

\section{Acknowledgements}

This work was supported by grants from the National Natural Science Foundation of China (No. 81302426, 31430045, 81470373, 81220108005 and 81300242) and the China Postdoctoral Science Foundation (2013 M540762).

\section{Author details}

${ }^{1}$ Cardiovascular Research Center, School of Medicine, Xi'an Jiaotong University, Xi'an 710061, China. ${ }^{2}$ Department of Public Health, School of Medicine, Xi'an Jiaotong University, Xi'an 710061, China. ${ }^{3}$ Nutrition and Food Safety Engineering Research Center of Shaanxi Province, School of Medicine, Xi'an Jiaotong University, Xi'an 710061, China. ${ }^{4}$ Institute of Cardiovascular Science, Peking University, Beijing 100191, China.

Received: 22 July 2014 Accepted: 27 November 2014

Published: 8 December 2014

\section{References}

1. Jonasson T, Ohlin AK, Gottsater A, Hultberg B, Ohlin H: Plasma homocysteine and markers for oxidative stress and inflammation in patients with coronary artery disease-a prospective randomized study of vitamin supplementation. Clin Chem Lab Med 2005, 43:628-634.

2. Malinow MR: Homocyst(e)ine and arterial occlusive diseases. $J$ Intern Med 1994, 236:603-617.

3. Clarke R, Daly L, Robinson K, Naughten E, Cahalane S, Fowler B, Graham I: Hyperhomocysteinemia: an independent risk factor for vascular disease. N Engl J Med 1991, 324:1149-1155.

4. Audelin MC, Genest JJ: Homocysteine and cardiovascular disease in diabetes mellitus. Atherosclerosis 2001, 159:497-511.

5. Werstuck GH, Lentz SR, Dayal S, Hossain GS, Sood SK, Shi YY, Zhou J, Maeda N, Krisans SK, Malinow MR, Austin RC: Homocysteine-induced endoplasmic reticulum stress causes dysregulation of the cholesterol and triglyceride biosynthetic pathways. J Clin Invest 2001, 107:1263-1273.

6. Herrmann W, Knapp JP: Hyperhomocysteinemia: a new risk factor for degenerative diseases. Clin Lab 2002, 48:471-481.

7. Polidori MC, Marvardi M, Cherubini A, Senin U, Mecocci P: Heart disease and vascular risk factors in the cognitively impaired elderly: implications for Alzheimer's dementia. Aging (Milano) 2001, 13:231-239.
8. Yan SK, Chang T, Wang H, Wu L, Wang R, Meng QH: Effects of hydrogen sulfide on homocysteine-induced oxidative stress in vascular smooth muscle cells. Biochem Biophys Res Commun 2006, 351:485-491.

9. Zou CG, Gao SY, Zhao YS, Li SD, Cao XZ, Zhang Y, Zhang KQ Homocysteine enhances cell proliferation in hepatic myofibroblastic stellate cells. J Mol Med (Berl) 2009, 87:75-84.

10. Ramakrishnan S, Sulochana KN, Lakshmi S, Selvi R, Angayarkanni N: Biochemistry of homocysteine in health and diseases. Indian J Biochem Biophys 2006, 43:275-283.

11. Takahashi T, Morita K, Akagi R, Sassa S: Heme oxygenase-1: a novel therapeutic target in oxidative tissue injuries. Curr Med Chem 2004, 11:1545-1561.

12. Shan Y, Pepe J, Lambrecht RW, Bonkovsky HL: Mapping of the chick heme oxygenase-1 proximal promoter for responsiveness to metalloporphyrins. Arch Biochem Biophys 2002, 399:159-166.

13. Shan Y, Pepe J, Lu TH, Elbirt KK, Lambrecht RW, Bonkovsky HL: Induction of the heme oxygenase-1 gene by metalloporphyrins. Arch Biochem Biophys 2000, 380:219-227

14. Ashino T, Sugiuchi J, Uehara J, Naito-Yamamoto Y, Kenmotsu S, Iwakura Y, Shioda S, Numazawa S, Yoshida T: Auranofin protects against cocaine-induced hepatic injury through induction of heme oxygenase- 1 . J Toxicol Sci 2011, 36:635-643.

15. Barikbin R, Neureiter D, Wirth J, Erhardt A, Schwinge D, Kluwe J, Schramm C Tiegs G, Sass G: Induction of heme oxygenase 1 prevents progression of liver fibrosis in Mdr2 knockout mice. Hepatology 2012, 55:553-562.

16. Kuramitsu K, Gallo D, Yoon M, Chin BY, Csizmadia E, Hanto DW, Otterbein LE: Carbon monoxide enhances early liver regeneration in mice after hepatectomy. Hepatology 2011, 53:2016-2026.

17. Mamiya T, Katsuoka F, Hirayama A, Nakajima O, Kobayashi A, Maher JM, Matsui H, Hyodo I, Yamamoto M, Hosoya T: Hepatocyte-specific deletion of heme oxygenase-1 disrupts redox homeostasis in basal and oxidative environments. Tohoku J Exp Med 2008, 216:331-339.

18. Schulz S, Wong RJ, Jang KY, Kalish F, Chisholm KM, Zhao H, Vreman HJ, Sylvester KG, Stevenson DK: Heme oxygenase-1 deficiency promotes the development of necrotizing enterocolitis-like intestinal injury in a newborn mouse model. Am J Physiol Gastrointest Liver Physiol 2013, 304:G991-G1001.

19. Alam J, Cook JL: How many transcription factors does it take to turn on the heme oxygenase-1 gene? Am J Respir Cell Mol Biol 2007, 36:166-174.

20. Fredenburgh LE, Perrella MA, Mitsialis SA: The role of heme oxygenase- 1 in pulmonary disease. Am J Respir Cell Mol Biol 2007, 36:158-165.

21. Andrews NC, Erdjument-Bromage $H$, Davidson MB, Tempst $\mathrm{P}$, Orkin SH: Erythroid transcription factor NF-E2 is a haematopoietic-specific basic-leucine zipper protein. Nature 1993, 362:722-728.

22. Alam J, Stewart D, Touchard C, Boinapally S, Choi AM, Cook JL: Nrf2, a Cap'n'Collar transcription factor, regulates induction of the heme oxygenase-1 gene. J Biol Chem 1999, 274:26071-26078.

23. Andrews NC, Kotkow KJ, Ney PA, Erdjument-Bromage H, Tempst $P$, Orkin SH: The ubiquitous subunit of erythroid transcription factor NF-E2 is a small basic-leucine zipper protein related to the $\mathrm{v}$-maf oncogene. Proc Natl Acad Sci U S A 1993, 90:11488-11492.

24. Liu C, Wang Q, Guo H, Xia M, Yuan Q, Hu Y, Zhu H, Hou M, Ma J, Tang Z, Ling W: Plasma S-adenosylhomocysteine is a better biomarker of atherosclerosis than homocysteine in apolipoprotein E-deficient mice fed high dietary methionine. J Nutr 2008, 138:311-315.

25. Luo X, Yang Y, Shen T, Tang X, Xiao Y, Zou T, Xia M, Ling W Docosahexaenoic acid ameliorates palmitate-induced lipid accumulation and inflammation through repressing NLRC4 inflammasome activation in HepG2 cells. Nutr Metab (Lond) 2012, 9:34.

26. Chan K, Han XD, Kan YW: An important function of Nrf2 in combating oxidative stress: detoxification of acetaminophen. Proc Natl Acad Sci U S A 2001, 98:4611-4616.

27. Xin HG, Zhang BB, Wu ZQ, Hang XF, Xu WS, Ni W, Zhang RQ, Miao XH: Treatment with baicalein attenuates methionine-choline deficient diet-induced non-alcoholic steatohepatitis in rats. Eur J Pharmacol 2014, 738:310-318.

28. Jia YH, Wang RQ, Mi HM, Kong LB, Ren WG, Li WC, Zhao SX, Zhang YG, Wu WJ, Nan YM, Yu J: Fuzheng Huayu recipe prevents nutritional fibrosing steatohepatitis in mice. Lipids Health Dis 2012, 11:45.

29. Huang XS, Chen HP, Yu HH, Yan YF, Liao ZP, Huang QR: Nrf2-dependent upregulation of antioxidative enzymes: a novel pathway for hypoxic 
preconditioning-mediated delayed cardioprotection. Mol Cell Biochem 2014, 385:33-41.

30. Chen CS, Tseng YT, Hsu YY, Lo YC: Nrf2-Keap1 antioxidant defense and cell survival signaling are upregulated by 17 beta-estradiol in homocysteinetreated dopaminergic SH-SY5Y cells. Neuroendocrinology 2013, 97:232-241.

31. Gao M, Singh A, Macri K, Reynolds C, Singhal V, Biswal S, Spannhake EW: Antioxidant components of naturally-occurring oils exhibit marked anti-inflammatory activity in epithelial cells of the human upper respiratory system. Respir Res 2011, 12:92.

32. Saw $C L$, Yang AY, Guo Y, Kong AN: Astaxanthin and omega-3 fatty acids individually and in combination protect against oxidative stress via the Nrf2-ARE pathway. Food Chem Toxicol 2013, 62:869-875.

33. Voelkel NF, Bogaard HJ, Al HA, Farkas L, Gomez-Arroyo J, Natarajan R: Antioxidants for the treatment of patients with severe angioproliferative pulmonary hypertension? Antioxid Redox Signal 2013, 18:1810-1817.

34. Kang SJ, You A, Kwak MK: Suppression of Nrf2 signaling by angiotensin II in murine renal epithelial cells. Arch Pharm Res 2011, 34:829-836.

35. Kratschmar DV, Calabrese D, Walsh J, Lister A, Birk J, Appenzeller-Herzog C, Moulin P, Goldring CE, Odermatt A: Suppression of the Nrf2-dependent antioxidant response by glucocorticoids and 11 beta-HSD1-mediated glucocorticoid activation in hepatic cells. PLoS One 2012, 7:e36774

36. Owuor ED, Kong AN: Antioxidants and oxidants regulated signal transduction pathways. Biochem Pharmacol 2002, 64:765-770.

37. Dhakshinamoorthy $S$, Jain AK, Bloom DA, Jaiswal AK: Bach1 competes with Nrf2 leading to negative regulation of the antioxidant response element (ARE)-mediated NAD(P)H:quinone oxidoreductase 1 gene expression and induction in response to antioxidants. J Biol Chem 2005, 280:16891-16900.

38. Shan Y, Lambrecht RW, Donohue SE, Bonkovsky HL: Role of Bach1 and Nrf2 in up-regulation of the heme oxygenase-1 gene by cobalt protoporphyrin. FASEB J 2006, 20:2651-2653.

39. Shan Y, Lambrecht RW, Ghaziani T, Donohue SE, Bonkovsky HL: Role of Bach-1 in regulation of heme oxygenase- 1 in human liver cells: insights from studies with small interfering RNAS. J Biol Chem 2004, 279:51769-51774.

40. Okita Y, Kamoshida A, Suzuki H, Itoh K, Motohashi H, Igarashi K, Yamamoto M, Ogami T, Koinuma D, Kato M: Transforming growth factor-beta induces transcription factors MafK and Bach1 to suppress expression of the heme oxygenase-1 gene. J Biol Chem 2013, 288:20658-20667.

41. Sun J, Hoshino H, Takaku K, Nakajima O, Muto A, Suzuki H, Tashiro S, Takahashi S, Shibahara S, Alam J, Taketo MM, Yamamoto M, Igarashi K: Hemoprotein Bach1 regulates enhancer availability of heme oxygenase-1 gene. EMBO J 2002, 21:5216-5224

42. Jyrkkanen HK, Kuosmanen $\mathrm{S}$, Heinaniemi M, Laitinen $H$, Kansanen $E_{\text {, }}$ Mella-Aho E, Leinonen H, Yla-Herttuala S, Levonen AL: Novel insights into the regulation of antioxidant-response-element-mediated gene expression by electrophiles: induction of the transcriptional repressor BACH1 by Nrf2. Biochem J 2011, 440:167-174.

43. Igarashi K, Sun J: The heme-Bach1 pathway in the regulation of oxidative stress response and erythroid differentiation. Antioxid Redox Signal 2006, 8:107-118.

44. Ogawa K, Sun J, Taketani S, Nakajima O, Nishitani C, Sassa S, Hayashi N, Yamamoto M, Shibahara S, Fujita H, Igarashi K: Heme mediates derepression of Maf recognition element through direct binding to transcription repressor Bach1. EMBO J 2001, 20:2835-2843.

45. Mani M, Golmohammadi T, Khaghani S, Zamani Z, Azadmanesh K, Meshkini R, Pasalar P: Homocysteine induces heme oxygenase-1 expression via transcription factor Nrf2 activation in HepG2 cell. Iran Biomed J 2013, 17:93-100

46. Sawle P, Foresti R, Green CJ, Motterlini R: Homocysteine attenuates endothelial haem oxygenase-1 induction by nitric oxide (NO) and hypoxia. FEBS Lett 2001, 508:403-406.

47. Niture SK, Khatri R, Jaiswal AK: Regulation of Nrf2-an update. Free Radic Biol Med 2014, 66:36-44

48. Jain AK, Jaiswal AK: Phosphorylation of tyrosine 568 controls nuclear export of Nrf2. J Biol Chem 2006, 281:12132-12142.

doi:10.1186/1743-7075-11-55

Cite this article as: Luo et al:: Homocysteine downregulates gene expression of heme oxygenase-1 in hepatocytes. Nutrition \& Metabolism 2014 11:55.

\section{Submit your next manuscript to BioMed Central and take full advantage of:}

- Convenient online submission

- Thorough peer review

- No space constraints or color figure charges

- Immediate publication on acceptance

- Inclusion in PubMed, CAS, Scopus and Google Scholar

- Research which is freely available for redistribution 